\title{
TOWARDS ENVIRONMENT MODELING BY AUTONOMOUS MOBILE SYSTEMS
}

\author{
Libor Přeučil, Petr Štěpán, Miroslav Kulich ${ }^{1}$, Roman Mázl \\ The Gertsner Laboratory for Intelligent Decision Making and Control \\ ${ }^{1}$ Center for Applied Cybernetics \\ Czech Technical University in Prague \\ \{preucil,stepan,kulich,mazl\}@labe.felk.cvut.cz
}

\begin{abstract}
The process of how to acquire knowledge about the operating environment is one of the most challenging problems that autonomous mobile robots must solve. The quality of the model depends on a number and the kind of sensors used and upon the precision the robot recovers its position. This paper introduces two crucial components of the map building procedure: the position localization based on data gathered from laser rangefinders and the deadreckoning system together with a novel method for map-building through data fusion from a monocular camera and a laser range-finder.
\end{abstract}

\section{INTRODUCTION}

In order to explore a working environment and to build a model of the environment a robot has to fulfill two fundamental tasks: (1) localize its position from observations and (2) recover the environment shape and structure. Both tasks are complementary and cannot be solved alone.

Local navigation systems of mobile robots typically provide sufficient accuracy only in a short-term periods, therefore independent localization technique has to be applied. The localization methods relying on range-measurements of the environment can be split into the following basic categories:

The main idea of landmark localization is to detect and match characteristic features (artificial or natural) in the environment from sensory inputs. (Leonard et al. 1991). The Markov localization evaluates a probability distribution over the space of possible robot states that represent positions (Fox et al. 1999).

The most promising sensor matching techniques compare raw or pre-processed sets of range-data obtained from sensors (actual scan) with a map or previously obtained sensor range-measurements (reference scan). These can be distinguished into point-to-point, point-to-line or line-to-line methods which compare particular types of measurements - or from these recovered point-based features as corners, line segments, etc. The comparison is done in a two-step process: building point-pair or feature-pair correspondences followed by translations and rotations to fit the actual and the reference scan. 
A critical part of the point-to-point approach is retrieval of the corresponding pairs of points what leads to preference of the point-to-line algorithms. The main idea is to approximate the reference scan by a list of lines or to match the actual scan with visible lines of the map (Gutmann et al. 1998). Similarly, method extension to line-to-line approach is straightforward and has been applied in the following (Chmelař et al. 2000).

Having solved the localization task, other sensors (which do not provide direct range measurements) can be incorporated to improve the robot navigation performance. Major attention is devoted in particular to integration of a monocular camera into the navigation process and world model build-up procedure. The task setup is thoroughly constrained to situations in which the robot operates on a plane and which covers a significant class of problems. The typical situations alike appear in indoor environments or even outdoors, where this condition is satisfied on the roads (Broggi, 1998).

Fusion of the camera data with the rangefinder data seems to be possible by applying occupancy grids (Elfes, 1989), (Menezes et al., 1994), the central part of the method introduced in the following. Similarly, knowing the robot position the range-finder data can be used to calibrate the probability profiles for the camera. The combination of diverse sensors like range-finders and monocular camera in the introduced approach leads to building more accurate 2-D maps of the environment.

\section{SYSTEM LOCALIZATION}

The localization problem, as mentioned above, is invoked by the need of knowing position and heading of the robot in the global coordinate system. This information determines correspondences between subsequent sensor measurements from different positions during robot mission and therefore enables us to create and efficiently update internal models (in this case maps - occupancy grids) of the environment.

\subsection{Range-Data Segmentation}

As range-finder measurements are in a form of distances to rigid obstacles in selected discrete directions, the processing of obstacle boundaries requires segmentation into point sets creating a particular boundary segment.

For the segmentation a recursive interval-splitting approach (Chmelař et al., 2000) selecting the candidate points for a straight-line boundary segment has been developed. The algorithm takes advantage of naturally angle-ordered points obtained from a laser range-finder. Evaluation of the maximum segment curvature criterion followed by application of a LSQ provides sets of points that optimally split the original boundary into particular segments.

The less important and possibly missegmented elements are discarded in a postprocessing filtration using heuristic rules (Chmelař et al., 2000), (Mázl et al., 2001).

\subsection{Correspondence Line Search}

A crucial part of the method is to find for each line from the actual scan a corresponding line from the reference scan (if exists). We say, that two lines correspond if and only if differences of their directions and positions are sufficiently small. To specify this, lets denote $x$ and $y$ lengths of the lines, $a, b, c, d$ distances 
between their vertices, and $\phi$ stands for the angle between lines, all satisfying the following expressions (the $\Phi_{M A X}$ and $K$ are preselected thresholds for the desired level of line similarity).

$$
\phi<\Phi_{\text {MAX }} \frac{\min (a, b)+\min (c, d)}{x+y}<K \text {, }
$$

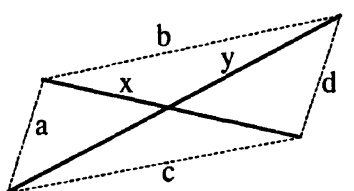

Figure 1 - Distance definition for evaluation of line-to-line similarity.

\subsection{Heading and Position Correction}

In this step, the heading correction is determined as the angular declination $\alpha$ between two scans. The leading idea is to evaluate a weighted sum of angular differences of each corresponding lines as:

$$
\alpha=\frac{\sum_{i=1}^{n} \phi_{i} w_{i}}{\sum_{i=1}^{n} w_{i}}
$$

where $n$ is a number of corresponding line pairs, $\phi_{i}$ is an angle between lines of the $i$-th pair, and $w_{i}$ is the weight of the $i$-th pair. The weight is defined as a product of the lines' lengths, which prefers pairs containing long lines to pairs of short lines.

In order to correct shifts in the position, we express each line of the reference scan in a standard form:

$$
a_{i} x+b_{i} y+c_{i}=0
$$

and each line of the actual scan is represented by its outer points $\left(\left[x_{i}{ }^{1}, y_{i}{ }^{1}\right],\left[x_{i}{ }^{2}, y_{i}{ }^{2}\right]\right)$, so that the shift $\left[p_{x}, p_{y}\right]$ of the actual scan can be determined by minimizing the following penalty function. The minimization problem itself can be solved via Nelder-Mead type simplex search.

$$
\min _{p_{x}, p_{y}} \sum_{i=1}^{n} \sum_{j=1}^{2}\left(a_{i}\left(x_{i}^{j}+p_{x}\right)+b_{i}\left(y_{i}^{j}+p_{y}\right)+c_{i}\right)^{2} .
$$

Illustrative results of position and heading correction based on processing of subsequent range-scan data are illustrated in the following figures.
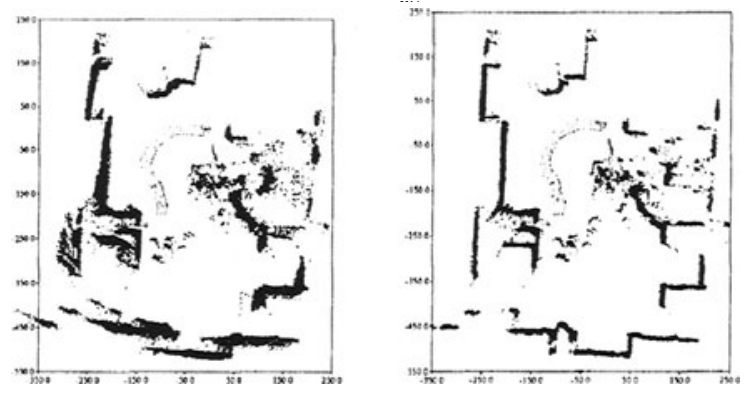

Figure 2 - Original Range-Data (Accumulated) with Errors in Heading and Shift (Left) and after Correction Using the Line-To-Line Approach (Right). 


\section{OCCUPANCY GRID FROM MONOCULAR CAMERA}

The occupancy grids belong to the most common low-level and sensor-based type model of the environment in robotics. The occupancy grid approach is very robust for fusion of noisy data and its application field has previously been limited to exclusive use with range sensors (originally designed for sonar data fusion).

The following shows, that under certain but reasonable circumstances the occupancy grid concept can also be used for fusion of monocular camera sensor.

A central assumption guaranteeing admissibility of the method is a robot operation on a flat ground-plane (floor). This assumption enables to determine all parameters of the robotics system. The parameters of the camera system can be calibrated on special images and afterwards used to detect obstacles in the robot surrounding.
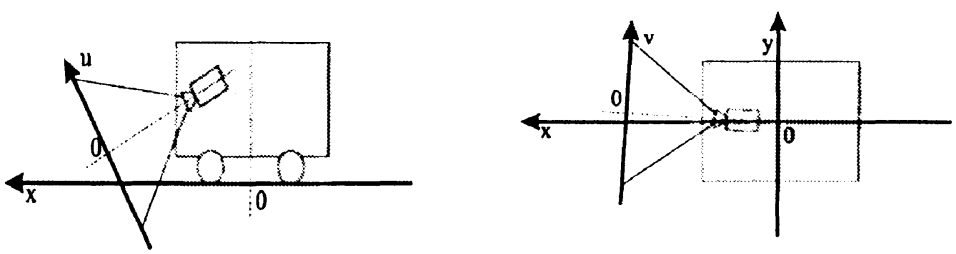

Figure 3 - Coordinate Systems for the Mobile Robot Onboard Camera

Our goal is to obtain 2-D map of the robot operating environment. It is necessary to find parameters of the robotic system to evaluate the transformation from the image coordinate system $(u, v)$ into the robot coordinate system $(x, y)$ - see figure 3 . It means to determine the projection function $f(u, v)=(x, y)$ (Šonka et al., 1998). The first step is a reduction of the radial distortion and decentering. The constants $u_{c}, v_{c}$ and $p_{1}, p_{2}, p_{3}$ can be determined from a calibration pattern according to the following equations:

$$
\begin{aligned}
& \text { dist }=\left(u-u_{c}\right)^{2}+\left(v-v_{c}\right)^{2} \\
& u^{\prime}=\left(u-u_{c}\right)\left(1+p_{1} \text { dist }+p_{2} \text { dist }^{2}+p_{3} \text { dist }^{3}\right) \\
& v^{\prime}=\left(v-v_{c}\right)\left(1+p_{1} \text { dist }+p_{2} \text { dist }^{2}+p_{3} \text { dist }^{3}\right)
\end{aligned}
$$

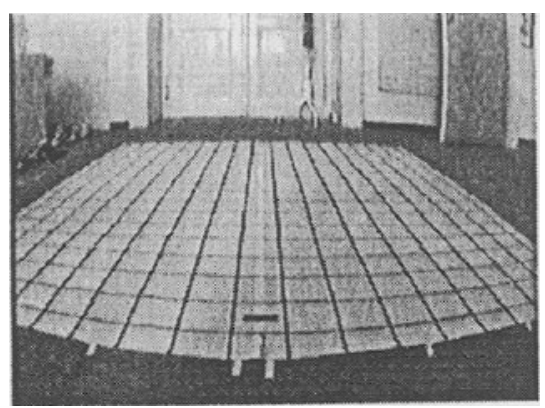

Figure 4 - The Calibration Pattern

To set the parameters of radial distortion and decentering the pattern in the Figure 4 was used. The image serves to detect the points from lines and the minimization of 
the square distance of these points to corresponding line was done. The results of this minimization are the $u_{c}, v_{c}$ and $p_{1,} p_{2,} p_{3}$ parameters.

The double $\left(u^{\prime}, v\right)$ determines the coordinates of the image after correction of the radial distortion. The next step is to detect the shift of the camera from the center of the robot $x_{\text {cam }} y_{\text {cam, }}$ and parameters of the plane that describes the floor of the environment, so $\Delta u, \Delta v, X$ and $Y$. The following equations describe the projective transformation:

$$
x_{0}=-\frac{\left(u^{\prime}-\Delta u\right) X}{v^{\prime}-\Delta v}+x_{c a m} \quad y_{0}=-\frac{Y}{v^{\prime}-\Delta v}+y_{c a m}
$$

A complete transformation is desired to correct the angular difference of the camera axis and the robot axis $\alpha$ as well as to obtain the coordinates in the robot world. Assume the robot position is $x_{r o b}, y_{r o b}, \omega_{r o b}$, the complete transformation can be described as:

$$
\begin{aligned}
& x=x_{r o b}+x_{0} \cos \left(\alpha+\omega_{r o b}\right)-y_{0} \sin \left(\alpha+\omega_{r o b}\right) \\
& y=y_{r o b}+x_{0} \sin \left(\alpha+\omega_{r o b}\right)+y_{0} \cos \left(\alpha+\omega_{r o b}\right),
\end{aligned}
$$

Eleven measurements of a reference box were done by camera and laser range finder to determine the above mentioned parameters. Each camera measurement represents four points (the borders of the box and two points on the front side) and the laser range finder provides the real position of this box. Minimization of the square distance from the real to the computed position in the camera image produces $x_{\text {cam }}$ $y_{c a m}, \Delta u, \Delta v, X, Y, \alpha$ for the robot system.

\subsection{Definition of the Probability Profiles}

Detection of the free space is done making-use of the color of the floor. Suppose that the color is different from the obstacle surface color. If the color of the floor is known the probability distribution of a free space can be defined. The occupancy grid (Elfes, 1989) is built using the Bayes' update formula:

$$
\begin{gathered}
P(O c c \mid R)=\frac{P(R \mid O c c) P(O c c)}{P(R \mid O c c) P(O c c)+P(R \mid E m p) P(E m p)} \\
\text { where: } \quad P(E m p)=1-P(O c c) \quad P(R \mid E m p)=1-P(R \mid O c c)
\end{gathered}
$$

The preceding formula defines new conditional probability, that the cell is occupied given measured color reading $R=(h, s, v)$. The probability depends on the old value $P(O c c)$ and the probability distribution $P(R \mid O c c)$. The probability distribution $P(R \mid O c c)$ can be represented as a difference of the measured color $R$ and the reference color of the floor in the hsv-space. The following can be used to compute the conditional probability depending on the reference color of the floor $\left(h_{r}, s_{r}, v_{r}\right)$ :

$$
\begin{aligned}
& \operatorname{dif}(x)=e^{-\frac{x^{2}}{2 \sigma^{2}}} \\
& P(R \mid O c c)=\operatorname{dif}\left(h-h_{r}\right) \operatorname{dif}\left(s-s_{r}\right) \operatorname{dif}\left(v-v_{r}\right),
\end{aligned}
$$

where $\sigma$ represents sensitivity of the difference function. 


\subsection{Calibration of the Probability Profiles with Range-Data}

If the color of the floor is similar to the color of the obstacles, the previous method fails. Another approach deriving the probability profiles uses definition of the profiles $P(R \mid O c c)$ with help of additional information about environment.

The additional information can be map of a training environment or combination of a sonar and laser data which can correctly measure the training environment. The probability profiles can be then calculated directly from the camera images. The free space and the border of the obstacle is determined from the range finder data or from the map of the environment in this case.

The matrices free and occ can be computed for a discrete hsv-space and they denote a number of cells with concrete color that are free or occupied, respectively. In our example, 10 images with different lighting conditions were used to create the matrixes free and occ with dimension $20 \times 20 \times 20$. For each pixel of the camera image, the occupancy of this pixel in the robot coordinate system was calculated. If the pixel with color $(h, s, v) \in<0,1\rangle^{3}$ represents a free space then the matrix value free $([20 h],[20 s],[20 v])$ is increased. If the pixel belongs to the occupied space, the matrix value $\operatorname{occ}([20 h],[20 s],[20 v])$ is increased. The probability profile $P(R \mid O c c)$ for reading $R=(h, s, v)$ is defined by the following formula:

$$
\begin{aligned}
& \text { if } \operatorname{free}\left(h^{\prime}, s^{\prime}, v^{\prime}\right)+o c c\left(h^{\prime}, s^{\prime}, v^{\prime}\right)>0 \text { then } \\
& P(R \mid O c c)=\frac{o c c\left(h^{\prime}, s^{\prime}, v^{\prime}\right)}{\operatorname{free}\left(h^{\prime}, s^{\prime}, v^{\prime}\right)+o c c\left(h^{\prime}, s^{\prime}, v^{\prime}\right)},
\end{aligned}
$$

otherwise: $P(R \mid O c c)=1$, where: $h^{\prime}=[20 h], s^{\prime}=[20 s], v^{\prime}=[20 v]$

The figure 5 shows original image from the onboard camera, the same image after coordinate transformation, where each pixel determines $5 \times 5 \mathrm{~cm}$ cell of the robot environment and finally the created occupancy grid using a probability profile matrix of dimension $20 \times 20 \times 20$.
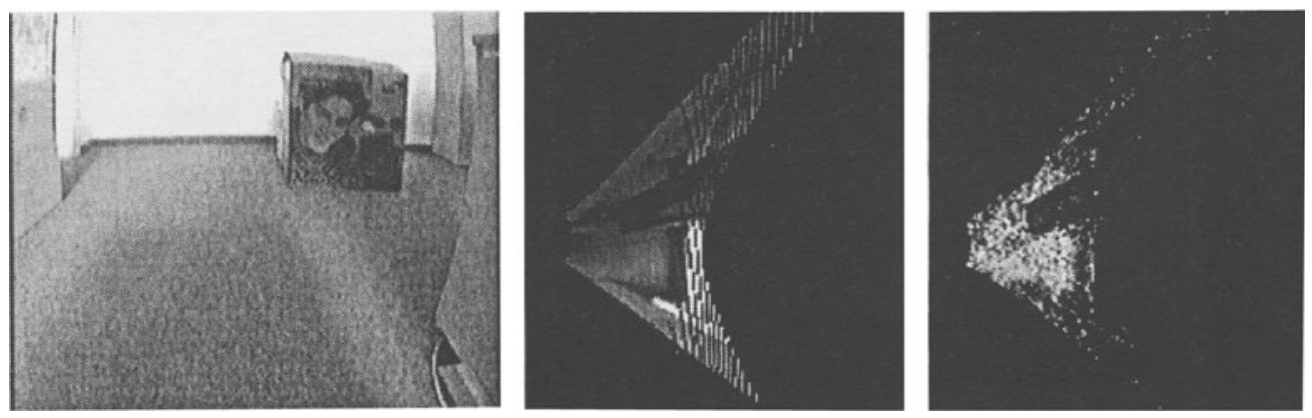

Figure 5 -The Original Onboard Camera Image (Left); after Transformation into the Floor Coordinate System (Middle) and the Final Occupancy Grid (Right).

The robot position is located on the left side of the picture a the camera aspect is oriented to the right in this case. White color stands for a free space as detected by the color probability profile of the floor plane. 


\subsection{Building the Final 2-D Map}

The final occupancy grid construction originates from the information about visibility. Unfortunately, it can often happen, that the color of the floor can appear at the obstacle too, even when the border of the obstacle is detected very well. Therefore, the final computation of the grid is done only within the interval from the position of the robot to the border of the grid. The accumulated probability is computed applying the following formula:

$$
P_{\text {new }}(O c c)=P(O c c) P_{\text {Acc }}(O c c)
$$

There are two possibilities how the accumulated probability $P_{A c c}(O c c)$ can be defined. The first one is simply the multiplication of the probabilities of all cells from the viewpoint. The second approach uses the Bayes formula for a recursive definition of this probability.

$$
P_{A c c^{\prime}}(O c c)=\frac{P(O c c) P_{A c c}(O c c)}{P(O c c) P_{A c c}(O c c)+(1-P(O c c))\left(1-P_{A c c}(O c c)\right)}
$$

The result of the Bayes update formula for the visibility occupancy grid is shown in the following figures.
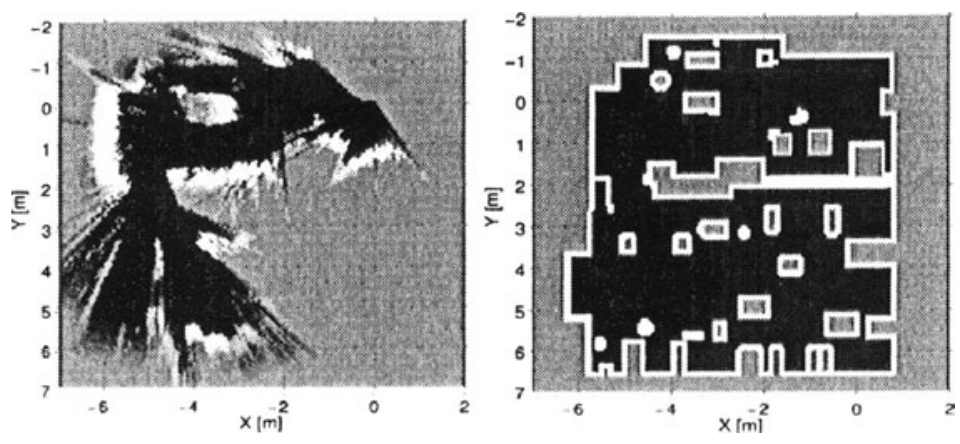

Figure 6- Final Occupancy Grid (Left) Created from Camera Data and the Obtained Environment Map after Segmentation (right).

The preceding figure illustrates an occupancy map created exclusively from camera data. The color model of the floor was created in a free space and the final map of a typical office environment can be obtained via image segmentation process (Sonka et al. 1998).

\section{CONCLUSION}

The problem of environment modeling for autonomous mobile systems - robots lasts still for a challenging field of many opened problems. The environment model recovery stands hand in hand with the localization problem. As there still does not exist any unified approach to reliable recovery of the environment models, the robustness is typically improved through data and model fusion from multiple sources.

The introduced novel method combines improved localization approach based on range-data from a LIDAR with a brand new usage of the occupancy grid approach. It has been illustrated, that under assumption that the robot operates in a 3D environment with a more or less uniform and flat ground-plane (e.g. floor, road, 
etc.), the occupancy grids can be extended towards data fusion from monocular camera. The introduced extension opens new possibilities in fusion in sensor-based models from more diverse sources like range-finder and monocular camera. This is expected to enable further improvement of the robustness and accuracy while building environment models in mobile robotics.

The early achievements of the described approach were experimentally verified with real data in real indoor environments. Respecting the limited space of this contribution, selected results were briefly illustrated in the running text above.

\section{ACKNOWLEDGMENT}

The work was supported by the Ministry of Education of the Czech Republic within the frame of the project "Decision-Making and Control for Manufacturing" number MSM 212300013, FRVS 2156/2002 and the Grant Agency of the Czech Republic 102/02/0641/A grants. The support of the Ministry of Education of the Czech Republic, under the Project No. LN00B096 to Miroslav Kulich, is also gratefully acknowledged.

\section{REFERENCES}

1. Broggi A, Bertozzi M, Fascioli A. An Extension to the Inverse Perspective Mapping to Handle Nonflat Roads. In: Proceedings of the IEEE International Conference on Intelligent Vehicles, Stuttgart, Germany, pp.305-309,1998.

2. Chmelał B, Płeucil L, Stexpán P. Range-data Based Position Localization and Refinement for a Mobile Robot. Proceedings of the 6th IFAC Symposium on Robot Control, Austria, Vienna, pp. 364-379, 2000.

3. Elfes A. Occupancy Grids: A Probabilistic Framework fo Robot Perception and Navigation. PhD thesis. Electrical and Computer Engineering Department/Robotics Institute. Carnegie-Mellon University, 1989.

4. Fox D, Burgard W, Thrun S. Markov Localization for Mobile Robots in Dynamic Environments. Journal of Artificial Intelligence, 11, pages 391-427, 1999.

5. Gutmann JS, Burgard W, Fox D, Konolige K. Experimental Comparison of Localization Methods. International Conference on Intelligent Robots and Systems, Victoria, B.C. , 1998.

6. Leonard JJ, Cox IJ, Durrant-Whyte HF. Dynamic Map Building for an Autonomous Mobile Robot. In Proc. IEEE Int. Workshop on Intelligent Robots and Systems, pages 89-96, July 1990. Also published in Autonomous Mobile Robots, edited by S. Iyengar and A. Elfes, Los Alamitos, CA: IEEE Computer Society Press, 1991.

7. Mázl R, Kulich M, Płeuxil L. Range Scan-based Localization Methods for Mobile Robots in Complex Environments. In: Proceedings: Intelligent Transportation Systems. IEEE Computer Society Press, New York, 2001, vol. 1, p. 280-285. ISBN 0-7803-7195-X

8. Menezes P, Arujo H, Dias J, Ribeiro MI. Obstacle Detection in Mobile Robots Using Sonar Data. In: Associacao Portuguesa de Controlo Automatico, Int. Conf. - Controlo 94, I.S.T., Lisbon, 1994.

9. Šonka M, HlaváC V, Boyle R. Image Processing, Analysis, and Machine Vision. Brooks/Cole Publishing Co., Boston, 1999, 800 p. ISBN 0-534-95393-X. 